\title{
A Simple Derivation of Burnashev's Reliability Function
}

\author{
Peter Berlin ${ }^{\dagger}$, Barış Nakiboğlu ${ }^{\ddagger}$, Bixio Rimoldi ${ }^{\dagger}$, Emre Telatar ${ }^{\dagger}$ \\ ${ }^{\dagger}$ School of Computer and Communication Sciences \\ Ecole Polytechnique Fédérale de Lausanne (EPFL) \\ CH-1015 Lausanne, Switzerland \\ ${ }^{\ddagger}$ Laboratory for Information and Decision Systems, \\ Department of EECS, Massachusetts Institute of Technology, \\ Cambridge, MA, USA \\ peter.berlindepfl.ch, nakib@mit.edu, \\ bixio.rimoldidepfl.ch, emre.telatardepfl.ch
}

\begin{abstract}
In a remarkable paper published in 1976, Burnashev determined the reliability function of variable length block codes over a discrete memoryless channel with feedback by providing a lower bound to the expected decoding time in terms of the size of the message set and the probability of error. We offer an alternative derivation of this lower bound. This derivation is simpler than the original and relates the quantities that appear in the bound to uncertainty reduction and binary hypothesis testing. Furthermore, the derivation closely parallels that of an upper bound by Yamamoto and Itoh.
\end{abstract}

\section{Index Terms}

Burnashev's error exponent, discrete memoryless channels (DMCs), feedback, variable-length communication

\section{INTRODUCTION}

It is well known [1] that the capacity of a discrete memoryless channel (DMC) is not increased by feedbackthe availability of the past channel outputs at the encoder. Nevertheless, feedback can simplify transmitter design. An example is the binary erasure channel, where the simplest possible transmitting strategy that makes use of feedback-repeating each information bit until it is received unerased-is not only capacity achieving, but does so with zero error probability and least possible delay.

Compared to capacity, the reliability function is a more refined measure of the quality of a channel. The familiar definition of the reliability function (or error exponent) is for the case of channels without feedback and block codes. Given a channel, one defines the reliability function as

$$
E(R)=\lim _{T \rightarrow \infty}-\frac{1}{T} \ln P_{e}\left(\left\lceil e^{R T}\right\rceil, T\right)
$$

where $P_{e}(M, T)$ is the smallest error probability that can be achieved by block codes with $M$ codewords and block length $T$. (Strictly speaking, in the absence of feedback it is not known if the limit exists for all rates; the sphere packing bound and its fellows upper bound the limsup, the random coding bound and its fellows lower bound the liminf.)

A natural way to quantify the advantage feedback brings to communication is to find the reliability function with feedback. Since in a communication system with feedback the decoding time $T$ may depend on the channel output 
sequence, $T$ is a random variable, and some care is needed to define the notions of rate and reliability function. Following Burnashev [2], we consider feedback block codes that have a fixed message set of size $M$ and define the rate as $R=(\ln M) / E[T]$. The reliability function is defined as

$$
E_{f}(R)=\lim _{\bar{T} \rightarrow \infty}-\frac{1}{\bar{T}} \ln P_{e, f}\left(\left\lceil e^{R \bar{T}}\right\rceil, \bar{T}\right)
$$

where $P_{e, f}(M, \bar{T})$ is the smallest error probability of a feedback code which transmits one of $M$ messages in expected time at most $\bar{T}$.

Burnashev shows that for a DMC of capacity $C$

$$
E_{f}(R)=E_{B}(R) \triangleq C_{1}(1-R / C), \quad 0 \leq R \leq C,
$$

where $C_{1}$ is determined by the two "most distinguishable" channel input symbols as

$$
C_{1}=\max _{x, x^{\prime}} D\left(p(\cdot \mid x) \| p\left(\cdot \mid x^{\prime}\right)\right) .
$$

Here, $p(\cdot \mid x)$ is the probability distribution of the output symbols of the channel when the input of the channel is $x$, and $D(\cdot \| \cdot)$ denotes the Kullback-Liebler divergence between two probability distributions. It is remarkable that this expression determines the reliability function exactly for all rates.

The main difficulty in showing that $E_{f}=E_{B}$ lies in proving that $E_{f} \leq E_{B}$. Burnashev does this by showing that

$$
E[T] \geq\left(\frac{\ln M}{C}-\frac{\ln P_{e}}{C_{1}}\right)(1+o(1))
$$

for any feedback communication scheme that transmit one of $M$ messages with error probability $P_{e}$. His proof is very instructive, but certainly not elementary. An example of Burnashev's exponent and the fixed length counterparts are shown (for a binary symmetric channel) in Fig. 1

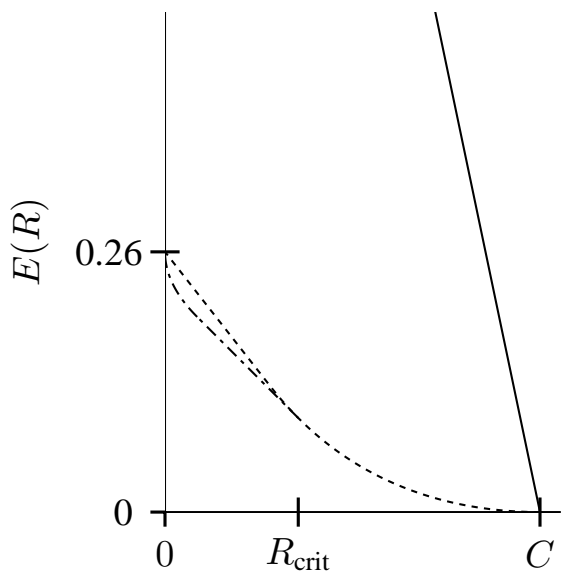

Fig. 1. Error exponents for a binary symmetric channel with crossover probability 0.1. Shown are the expurgated exponent (dot-dashed line), sphere packing and straight line bound (dotted line) and Burnashev's exponent (solid line) for rates from zero to capacity.

In contrast, Yamamoto and Itoh [3] have proposed and analyzed a scheme that asymptotically achieves Burnashev's exponent $E_{B}$. The analysis of this scheme is elementary. It is also instructive to observe that the two additive terms on the right of (3) are accounted for in two distinct transmission phases, which may be appropriately called the communication and confirmation phases. In the communication phase the message is encoded using a block code and transmitted over the channel. The decoder then makes a tentative decision about the message and communicates it to the encoder via the feedback channel. In the confirmation phase the encoder confirms a correct estimate by sending an ACK and denies an incorrect estimate with a NACK. ACKs and NACKs are sent via a 
repetition code. The decoder performs a binary hypothesis test to decide if an ACK or a NACK was transmitted. If an ACK is decoded, the tentative decision becomes final and the process stops (leaving the system free to restart with a new message). Otherwise, the process restarts with the same message. The overhead caused by the process restarting is negligible if the tentative decision is correct and confirmed successfully most of the time. For this to happen, the communication phase must make at least roughly $(\ln M) / C$ channel uses. The probability that the final decision is incorrect is essentially determined by the probability that a NACK is decoded as an ACK. Using Stein's lemma, one needs $-\left(\ln P_{e}\right) / C_{1}$ channel uses to achieve error probability $P_{e}$. The sum of $(\ln M) / C$ and $-\left(\ln P_{e}\right) / C_{1}$ is precisely the limit of the right hand side of (3).

The conceptual simplicity of the two-phase scheme proposed by Yamamoto and Itoh and the match between those two phases and the two terms on the right of (3) make one feel that the two phases are somewhat fundamental in achieving Burnashev's exponent with feedback. It is with this in mind that we have been looking for a simple converse that makes similar connections. The goal of this paper is to present such a converse. For the proof of the main result, stated as a theorem, we use two intermediate results called lemmas. All other results are called propositions.

\section{Channel Model and Variable Length Codes as Trees}

We consider a discrete memoryless channel, with channel input alphabet $\mathcal{X}$, output alphabet $\mathcal{Y}$, both finite, and transition probabilities $p(y \mid x)$. We will denote the channel input and output symbols at time $n$ by $X_{n}$ and $Y_{n}$, and denote the corresponding vectors $\left(X_{1}, X_{2}, \ldots, X_{n}\right)$ and $\left(Y_{1}, Y_{2}, \ldots, Y_{n}\right)$ by $X^{n}$ and $Y^{n}$, respectively. A perfect causal feedback link is available, i.e., the encoder at time $n$ knows $y^{n-1}$. (Following common practice, random variables are represented by capital letters and their realizations are denoted by the corresponding lowercase letters.)

We will assume, without loss of generality, that the channel has no "useless outputs symbols", i.e., no symbols $y$ for which $p(y \mid x)=0$ for every $x$. Note that for channels for which $C_{1}$ is infinite, the lower bound to the expected decoding time is a restatement of the fact that feedback does not increase capacity. We will therefore restrict our attention to channels for which $C_{1}<\infty$. For such channels, $p(y \mid x)>0$ for every $x$ and $y$; if not, there exists an $x$ and $y$ for which $p(y \mid x)=0$. Since $y$ is reachable from some input, there also exists an $x^{\prime}$ for which $p\left(y \mid x^{\prime}\right)>0$. But then $D\left(p\left(\cdot \mid x^{\prime}\right) \| p(\cdot \mid x)\right)=\infty$ contradicting the finiteness of $C_{1}$. The fact that both $\mathcal{X}$ and $\mathcal{Y}$ are finite sets lets us further conclude that for the channels of interest to this paper, there is a $\lambda>0$ for which $p(y \mid x) \geq \lambda$ for every $x$ and $y$.

A variable length block code is defined by two maps: the encoder and the decoder. The encoder ${ }^{1}$ functions $f_{n}(\cdot, \cdot): \mathcal{W} \times \mathcal{Y}^{n-1} \rightarrow \mathcal{X}$, where $\mathcal{W}$ is the set of all possible messages, determine the channel input $X_{n}=$ $f_{n}\left(W, Y^{n-1}\right)$ based on the message $W$ and on past channel outputs $Y^{n-1}$. The decoder function $\hat{W}(\cdot): \mathcal{Z} \rightarrow \mathcal{W}$, where $\mathcal{Z}$ is the receiver observation space until the decoding time $T$, i.e., $Y^{T}$ takes values in $\mathcal{Z}$. The decoding time $T$ should be a Markov stopping time with respect to the receiver observation $Y^{n}$.

The observation space $\mathcal{Z}$ is a collection of channel output sequences and for a DMC with feedback the length of these sequences may vary. (The length of the channel input itself may depend on the channel realization). Nevertheless, these sequences have the property of being prefix-free (otherwise the decision to stop would require knowledge of the future) and complete in the sense that every infinite sequence of channel outputs has a prefix in $\mathcal{Z}$ (otherwise there will be cases when a decision is never made). Thus, $\mathcal{Z}$ can be represented as the leaves of a complete $|\mathcal{Y}|$-ary tree $\mathcal{T}$ (complete in the sense that each intermediate node has $|\mathcal{Y}|$ descendants) with expected depth $E[T]<\infty$. Note that the decision time $T$ is simply the first time the sequence $Y_{1}, Y_{2}, \ldots$ of channel outputs hits a leaf of $\mathcal{T}$. Furthermore we may label each leaf of $\mathcal{T}$ with the message estimate produced by the decoder when that leaf is reached. This way the decoder is completely specified by the labelled tree $\mathcal{T}$. The message statistics, the code, and the channel determine a probability measure on the tree $\mathcal{T}$.

\footnotetext{
${ }^{1}$ For clarity of exposition we will only treat deterministic coding strategies here. Randomized strategies may be included without significant modification to the core of the proof.
} 


\section{BinARy Hypothesis Testing With FeEdBACK}

The binary case will play a key role in our main proof. In this section we assume that the message set contains only two elements that we will arbitrarily denote by $A$ and $N$ (ACK and NACK, respectively). We denote by $\mathcal{Q}_{A}$ and $\mathcal{Q}_{N}$ the corresponding probability distributions on the leaves of $\mathcal{T}$.

The following proposition bounds the Kullback-Leibler distance $D\left(\mathcal{Q}_{A} \| \mathcal{Q}_{N}\right)$ in terms of the expected depth $E[T \mid A]$ of the tree under $\mathcal{Q}_{A}$. It will be used in the main result of this section to bound the error probability of binary hypothesis testing with feedback. The reader familiar with Stein's Lemma [4] will not be surprised by the fact that the Kullback-Leibler distance $D\left(\mathcal{Q}_{A} \| \mathcal{Q}_{N}\right)$ plays a key role in binary hypothesis testing with feedback.

Proposition 1: For any binary hypothesis testing scheme for a channel with feedback

$$
D\left(\mathcal{Q}_{A} \| \mathcal{Q}_{N}\right) \leq C_{1} E[T \mid A]
$$

where $T$ is the decision time, $E[T]<\infty$, and $E[\cdot \mid A]$ denotes expectation under the measure $\mathcal{Q}_{A}$, i.e., conditioned on hypothesis $A$.

Proof: Let

$$
V_{n}=\ln \frac{\mathcal{Q}_{A}\left(Y_{1}, \ldots, Y_{n}\right)}{\mathcal{Q}_{N}\left(Y_{1}, \ldots, Y_{n}\right)}
$$

so that $D\left(\mathcal{Q}_{A} \| \mathcal{Q}_{N}\right)=E\left[V_{T} \mid A\right]$, and the proposition is equivalent to the statement $E\left[V_{T}-C_{1} T \mid A\right] \leq 0$. Observe that

$$
V_{n}=\sum_{k=1}^{n} U_{k} \quad \text { where } U_{k}=\ln \frac{\mathcal{Q}_{A}\left(Y_{k} \mid Y^{k-1}\right)}{\mathcal{Q}_{N}\left(Y_{k} \mid Y^{k-1}\right)}
$$

Note now that

$$
\begin{aligned}
E\left[U_{k} \mid A, Y^{k-1}\right] & =E\left[\ln \frac{\mathcal{Q}_{A}\left(Y_{k} \mid Y^{k-1}\right)}{\mathcal{Q}_{N}\left(Y_{k} \mid Y^{k-1}\right)} \mid A, Y^{k-1}\right] \\
& =E\left[\ln \frac{\mathcal{Q}_{A}\left(Y_{k} \mid X_{k}=f_{k}\left(A, Y^{k-1}\right), Y^{k-1}\right)}{\mathcal{Q}_{N}\left(Y_{k} \mid X_{k}=f_{k}\left(N, Y^{k-1}\right), Y^{k-1}\right)} \mid A, X_{k}=f_{k}\left(A, Y^{k-1}\right), Y^{k-1}\right] \\
& =\sum_{y \in \mathcal{Y}} \operatorname{Pr}\left\{Y_{k}=y \mid X_{k}=f_{k}\left(A, Y^{k-1}\right)\right\} \ln \frac{\operatorname{Pr}\left\{Y_{k}=y \mid X_{k}=f_{k}\left(A, Y^{k-1}\right)\right\}}{\operatorname{Pr}\left\{Y_{k}=y \mid X_{k}=f_{k}\left(N, Y^{k-1}\right)\right\}} \\
& \leq C_{1} .
\end{aligned}
$$

Consequently, $V_{n}-n C_{1}$ is a supermartingale under hypothesis $A$. Observe that the existance of a $\lambda>0$ for which $p(y \mid x)>\lambda$ for all $x, y$ implies that $\left|U_{k}\right|<\ln \frac{1}{\lambda}$. We can now use Doob's Optional-Stopping Theorem [5] to conclude that $E\left[V_{T}-C_{1} T \mid A\right] \leq 0$.

We can apply Proposition 1 to find a lower bound on the error probability of a binary hypothesis testing problem with feedback. The bound is expressed in terms of the expected decision time.

Lemma 1: The error probability of a binary hypothesis test performed across a DMC with feedback and variable length codes is lower bounded by

$$
P_{e} \geq \frac{\min \left\{P_{A}, P_{N}\right\}}{4} e^{-C_{1} E[T]}
$$

where $P_{A}$ and $P_{N}$ are the a priori probabilities.

Proof: Each different decoding method corresponds to a tree where each leaf $Y^{T}$ is associated with a decoded message $\hat{W}\left(Y^{T}\right)$. Thus we can partition the leaves into two sets corresponding to the two messages.

$$
\begin{aligned}
& \mathcal{S}=\left\{y^{T}: \hat{W}\left(y^{T}\right)=A\right\} \\
& \overline{\mathcal{S}}=\left\{y^{T}: \hat{W}\left(y^{T}\right) \neq A\right\}
\end{aligned}
$$


where $\mathcal{S}$ is the decision region for message $A$.

The log sum inequality [4], [6] (or data processing lemma for divergence) implies

$$
D\left(\mathcal{Q}_{A} \| \mathcal{Q}_{N}\right) \geq \mathcal{Q}_{A}(\mathcal{S}) \ln \frac{\mathcal{Q}_{A}(\mathcal{S})}{\mathcal{Q}_{N}(\mathcal{S})}+\mathcal{Q}_{A}(\overline{\mathcal{S}}) \ln \frac{\mathcal{Q}_{A}(\overline{\mathcal{S}})}{\mathcal{Q}_{N}(\overline{\mathcal{S}})}
$$

By Proposition 11 $C_{1} E[T \mid A] \geq D\left(\mathcal{Q}_{A} \| \mathcal{Q}_{N}\right)$, thus (4) can be re-arranged to give

$$
C_{1} E[T \mid A] \geq-\mathcal{Q}_{A}(\mathcal{S}) \ln \mathcal{Q}_{N}(\mathcal{S})-\mathrm{h}\left(\mathcal{Q}_{A}(\overline{\mathcal{S}})\right),
$$

where $h(\cdot)$ is the binary entropy function. Writing the overall probability of error in terms of marginal error probabilities yields

$$
P_{e}=P_{N} \mathcal{Q}_{N}(\mathcal{S})+P_{A} \mathcal{Q}_{A}(\overline{\mathcal{S}})
$$

which allows us to bound $\mathcal{Q}_{N}(\mathcal{S})$ as

$$
\mathcal{Q}_{N}(\mathcal{S}) \leq \frac{P_{e}}{\min \left\{P_{A}, P_{N}\right\}} .
$$

Substituting back into (5) yields a bound on the expected depth of the decision tree conditioned on $A$ just in terms of $\mathcal{Q}_{A}$ and the a priori message probabilities

$$
C_{1} E[T \mid A] \geq-\mathcal{Q}_{A}(\mathcal{S}) \ln \frac{P_{e}}{\min \left\{P_{A}, P_{N}\right\}}-\mathrm{h}\left(\mathcal{Q}_{A}(\overline{\mathcal{S}})\right) .
$$

Following identical steps with the roles of $A$ and $N$ swapped yields

$$
C_{1} E[T \mid N] \geq-\mathcal{Q}_{N}(\overline{\mathcal{S}}) \ln \frac{P_{e}}{\min \left\{P_{A}, P_{N}\right\}}-\mathrm{h}\left(\mathcal{Q}_{N}(\mathcal{S})\right) .
$$

We can now average both sides of (6) and (7) by weighting with the corresponding a priori probabilities. If we do so and use the fact that $P_{A} \mathcal{Q}_{A}(\mathcal{S})+P_{N} \mathcal{Q}_{N}(\overline{\mathcal{S}})$ is the probability of making the correct decision, $P_{A} \mathcal{Q}_{A}(\overline{\mathcal{S}})+P_{N} \mathcal{Q}_{N}(\mathcal{S})$ the probability of making an error, and use the concavity of the binary entropy function, we obtain the following unconditional bound on the depth of the decision tree

$$
\begin{aligned}
C_{1} E[T] & \geq-\left(1-P_{e}\right) \ln \frac{P_{e}}{\min \left\{P_{A}, P_{N}\right\}}-\mathrm{h}\left(P_{e}\right) \\
& \geq-\ln P_{e}-2 \mathrm{~h}\left(P_{e}\right)+\ln \min \left\{P_{A}, P_{N}\right\} \\
& \geq-\ln P_{e}-2 \ln 2+\ln \min \left\{P_{A}, P_{N}\right\} .
\end{aligned}
$$

Solving for $P_{e}$ completes the proof,

$$
P_{e} \geq \frac{\min \left\{P_{A}, P_{N}\right\}}{4} e^{-C_{1} E[T]}
$$

It is worthwhile pointing out why the factor $\min \left\{P_{A}, P_{N}\right\}$ arises: if one of the hypotheses has small a priori probability, one can always achieve an equally small error probability by always deciding for the other hypothesis, irrespective of the channel observations.

\section{Expected Tree DePth AND Channel CAPACity}

Given the channel observations $y^{n}$, one can calculate the a posteriori probability $p_{W \mid Y^{n}}\left(w \mid y^{n}\right)$ of any message $w \in \mathcal{W}$. Recall that a maximum a posteriori (MAP) decoder asked to decide at time $n$ when $Y^{n}=y^{n}$ will chose (one of) the message(s) that has the largest a posteriori probability $p_{\max }=\max _{w} p_{W \mid Y^{n}}\left(w \mid y^{n}\right)$. The probability of error will then be $P_{e}\left(y^{n}\right)=1-p_{\text {max }}$. Similarly, we can define the probability of error of a MAP decoder for each leaf of the observation tree $\mathcal{T}$. Let us denote by $P_{e}\left(y^{T}\right)$ the probability of error given the observation $y^{T}$. The unconditional probability of error is then $P_{e}=E\left[P_{e}\left(Y^{T}\right)\right]$. 
We can define a stopping time $\tau$ as the first time that the error probability goes below $\delta$, if this happens before $T$, and as $T$ otherwise:

$$
\tau=\min \left\{n: n=T \text { or } P_{e}\left(y^{n}\right) \leq \delta\right\}
$$

If $P_{e}\left(Y^{\tau}\right)$ exceeds $\delta$, then we are certain that $\tau=T$. The probability of this event can be upper bounded by

$$
\operatorname{Pr}\left\{P_{e}\left(Y^{\tau}\right)>\delta\right\} \leq \operatorname{Pr}\left\{P_{e}\left(Y^{T}\right)>\delta\right\} \leq \frac{P_{e}}{\delta},
$$

where the second inequality is an application of Markov's inequality.

Given a particular realization $y^{n}$, we will denote the entropy of the a posteriori distribution $p_{W \mid Y^{n}}\left(\cdot \mid y^{n}\right)$ as $h\left(y^{n}\right)$. Then $E\left[h\left(Y^{n}\right)\right]=H\left(W \mid Y^{n}\right)$. From Fano's inequality it follows that if $P_{e}\left(y^{\tau}\right) \leq \delta \leq \frac{1}{2}$, then

$$
h\left(y^{\tau}\right) \leq \mathrm{h}(\delta)+\delta \ln |\mathcal{W}|
$$

The expected value of $h\left(y^{\tau}\right)$ can be bounded by conditioning on the event $P_{e}\left(y^{\tau}\right) \leq \delta$ and its complement then applying (10) and then (9) as follows

$$
\begin{aligned}
E\left[h\left(Y^{\tau}\right)\right] & =E\left[h\left(Y^{\tau}\right) \mid P_{e}\left(Y^{\tau}\right) \leq \delta\right] \operatorname{Pr}\left\{P_{e}\left(Y^{\tau}\right) \leq \delta\right\}+E\left[h\left(Y^{\tau}\right) \mid P_{e}\left(Y^{\tau}\right)>\delta\right] \operatorname{Pr}\left\{P_{e}\left(Y^{\tau}\right)>\delta\right\} \\
& \left.\leq(\mathrm{h}(\delta)+\delta \ln |\mathcal{W}|) \operatorname{Pr}\left\{P_{e}\left(Y^{\tau}\right) \leq \delta\right\}+(\ln |\mathcal{W}|) \operatorname{Pr}\left\{P_{e}\left(Y^{\tau}\right)>\delta\right)\right\} \\
& \leq \mathrm{h}(\delta)+\left(\delta+\frac{P_{e}}{\delta}\right) \ln |\mathcal{W}|
\end{aligned}
$$

This upper bound on the expected posterior entropy at time $\tau$ can be turned into a lower bound on the expected value of $\tau$ by using the channel capacity as an upper bound to the expected change of entropy. This notion is made precise by the following lemma,

Lemma 2: For any $0<\delta \leq \frac{1}{2}$

$$
E[\tau] \geq\left(1-\delta-\frac{P_{e}}{\delta}\right) \frac{\ln |\mathcal{W}|}{C}-\frac{\mathrm{h}(\delta)}{C}
$$

Proof: Observe that $h\left(Y^{n}\right)+n C$ is a submartingale (an observation already made in [2, Lemma 2]). To see this,

$$
\begin{aligned}
E\left[h\left(Y^{n-1}\right)-h\left(Y^{n}\right) \mid Y^{n-1}=y^{n-1}\right] & =I\left(W ; Y_{n} \mid Y^{n-1}=y^{n-1}\right) \\
& \stackrel{(a)}{\leq} I\left(X_{n} ; Y_{n} \mid Y^{n-1}=y^{n-1}\right) \\
& \leq C
\end{aligned}
$$

where $(a)$ follows from the data processing inequality and the fact that $X_{n}$ is a function of $W$ given $Y^{n-1}=y^{n-1}$. Hence $h\left(Y^{n}\right)+n C$ is indeed a submartingale. Since $h\left(y^{n}\right)$ is bounded between 0 and $\ln |\mathcal{W}|$ for all $n$, and the expected stopping time $E[\tau] \leq E[T]<\infty$, Doob's Optional-Stopping Theorem [5] allows us to conclude that at time $\tau$ the expected value of the submartingale must be above the starting point $\ln |\mathcal{W}|$. Hence

$$
\begin{aligned}
\ln |\mathcal{W}|=h\left(y^{0}\right) & \leq E\left[h\left(Y^{\tau}\right)+\tau C\right] \\
& =E\left[h\left(Y^{\tau}\right)\right]+E[\tau] C \\
& \leq \mathrm{h}(\delta)+\left(\delta+\frac{P_{e}}{\delta}\right) \ln |\mathcal{W}|+E[\tau] C
\end{aligned}
$$

Solving for $E[\tau]$ yields

$$
E[\tau] \geq\left(1-\delta-\frac{P_{e}}{\delta}\right) \frac{\ln |\mathcal{W}|}{C}-\frac{\mathrm{h}(\delta)}{C}
$$




\section{BURNASHEV's LOWER BOUND}

In this section we will combine the two bounds we have established in the preceding sections to obtain a bound on the overall expected decoding time. Lemma 2 provides a lower bound on $E[\tau]$ as a function of $\delta$ and $P_{e}$. We will then show that a properly constructed binary hypothesis testing problem allows us to use Lemma 1 to bound the probability of error in terms of $E\left[T-\tau \mid Y^{\tau}\right]$. This in turn will lead us to the final bound on $E[T]$.

The next proposition states that a new channel output symbol can not change the a posteriori probability by more than some constant factor when $C_{1}$ is finite.

Proposition 2: $C_{1}<\infty$ implies

$$
\lambda p\left(w \mid y^{n-1}\right) \leq p\left(w \mid y^{n}\right) \leq \frac{p\left(w \mid y^{n-1}\right)}{\lambda}
$$

where $0<\lambda=\min _{x, y} p(y \mid x) \leq \frac{1}{2}$.

Proof: Using Bayes' rule, the posterior may be written recursively as

$$
p\left(w \mid y^{n}\right)=p\left(w \mid y^{n-1}\right) \frac{p\left(y_{n} \mid x_{n}=f_{n}\left(w, y^{n-1}\right)\right)}{p\left(y_{n} \mid y^{n-1}\right)} .
$$

The quotient may be upper and lower bounded using $1 \geq p\left(y_{n} \mid x_{n}=f_{n}\left(w, y^{n-1}\right)\right) \geq \lambda$ and $1 \geq p\left(y_{n} \mid y^{n-1}\right) \geq \lambda$, which yields the statement of the proposition.

Our objective is to lowerbound the probability of error. The key idea is that a binary decision such as deciding whether or not $W$ lies in a specified set $\mathcal{G}$ can be done more reliably than producing an estimate of the value of $W$. Indeed, if $\hat{W}$ is the message estimate produced by the decoder, we can decide that $W \in \mathcal{G}$ iff $\hat{W} \in \mathcal{G}$. This binary decision is always correct when the decoder makes the correct decision about $W$. Hence the probability of error of this (not necessarily optimal) binary decision rule cannot exceed the probability of error of the decoder. The probability of error for the binary decision rule may be lowerbounded by Lemma 1 The details of this follow.

For every realization of $Y^{\tau}$ we can divide the message set into two parts, say $\mathcal{G}$ and its complement $\mathcal{W} \backslash \mathcal{G}$, in such a way that each of them has a posteriori probability greater then $\lambda \delta$. The rest of this paragraph explains how this is possible. First observe that at time $\tau-1$ the a posteriori probability of every message is smaller than $1-\delta$. This implies that the sum of the a posteriori probabilities of any set of $(|\mathcal{W}|-1)$ messages is greater than $\delta$ at time $\tau-1$ and greater than $\delta \lambda$ at time $\tau$. In particular, $P_{e}\left(y^{\tau}\right) \geq \lambda \delta$. Consider first the case when $P_{e}\left(y^{\tau}\right) \leq \delta$. We may let $\mathcal{G}$ be the set that consists of the MAP decision made at time $\tau$. Then $\operatorname{Pr}\{\mathcal{G}\} \geq 1-\delta \geq 1 / 2 \geq \lambda \delta$. As argued above its complement also has probability greater than $\lambda \delta$, thus this partition satisfies the required condition for this case. If however $P_{e}\left(y^{\tau}\right)>\delta$, we know for sure that the a posteriori probability of each message is smaller than $1-\delta$. We form the set $\mathcal{G}$ by starting with the empty set and adding messages in an arbitrary fashion until we exceed the threshold $\delta / 2$. The threshold will be exceeded by at most $1-\delta$. Thus $\operatorname{Pr}\{\mathcal{G}\} \leq 1-\delta / 2$, i.e., $\operatorname{Pr}\{\overline{\mathcal{G}}\} \geq \delta / 2$. By construction $\operatorname{Pr}\{\mathcal{G}\} \geq \delta / 2$ thus $\min (\operatorname{Pr}\{\mathcal{G}\}, \operatorname{Pr}\{\overline{\mathcal{G}}\}) \geq \delta / 2 \geq \lambda \delta$.

Lemma 1 can be applied with $A=\mathcal{G}$ and $N=\overline{\mathcal{G}}$ to lower bound the probability of error of the above binary hypothesis testing problem, and hence also the true probability of error as follows,

$$
\operatorname{Pr}\left\{\hat{W}\left(Y^{T}\right) \neq W \mid Y^{\tau}\right\} \geq \frac{\lambda \delta}{4} e^{-C_{1} E\left[T-\tau \mid Y^{\tau}\right]} .
$$

Taking the expectation of the above expression yields the unconditional probability of error

$$
P_{e}=E\left[\operatorname{Pr}\left\{\hat{W}\left(Y^{T}\right) \neq W \mid Y^{\tau}\right\}\right] \geq E\left[\frac{\lambda \delta}{4} e^{-C_{1} E\left[T-\tau \mid Y^{\tau}\right]}\right]
$$

Using the convexity of $e^{-x}$ and Jensen's inequality, we obtain

$$
P_{e} \geq \frac{\lambda \delta}{4} e^{-C_{1} E[T-\tau]}
$$


Solving for $E[T-\tau]$ yields

$$
E[T-\tau] \geq \frac{-\ln P_{e}-\ln 4+\ln (\lambda \delta)}{C_{1}}
$$

Combing Lemma 2 and $[11$ yields:

Theorem 1: The expected decoding time $T$ of any variable length code for a DMC used with feedback is lower bounded by

$$
E[T] \geq\left(1-\delta-\frac{P_{e}}{\delta}\right) \frac{\ln |\mathcal{W}|}{C}+\frac{-\ln P_{e}}{C_{1}}-\frac{\mathrm{h}(\delta)}{C}+\frac{\ln (\lambda \delta)-\ln 4}{C_{1}},
$$

where $|\mathcal{W}|$ is the cardinality of the message set, $P_{e}$ the error probability, $\lambda=\min _{x \in \mathcal{X}, y \in \mathcal{Y}} p(y \mid x)$, and $\delta$ is any number that satisfies $0<\delta \leq 0.5$.

\section{CONCLUSION}

We have presented a new derivation of Burnashev's asymptotically tight lower bound to the average delay needed for a target error probability when a message is communicated across a DMC used with (channel output) feedback. Our proof is simpler than the original, yet provides insight by clarifying the role played by the quantities that appear in the bound. Specifically, from the channel coding theorem we expect it to take roughly $\frac{\ln |\mathcal{W}|}{C}$ channel uses to reduce the probability of error of a MAP decision to some small (but not too small) value. At this point we can partition the message set in two subsets, such that neither subset has too small an a posteriori probability. From now on it takes (asymptotically) $-\frac{\ln P_{e}}{C_{1}}$ channel uses to decide with probability of error $P_{e}$ which of the two sets contains the true message. It takes at least as many channel uses to decide which message was selected and incur the same error probability.

For obvious reasons we may call the two phases the communication and the binary hypothesis testing phase, respectively. These two phases exhibit a pleasing similarity to the communication and confirmation phase of the optimal scheme proposed and analyzed by Yamamoto and Itoh in [3]. The fact that these two phases play a key role in proving achievability as well as in showing that one can not do better suggests that they are an intrinsic component of an optimal communication scheme using variable length block codes over DMCs with feedback.

\section{ACKNOWLEDGEMENT}

The authors would like to thank R. G. Gallager for his help in pointing out a error in an earlier version of this paper.

\section{REFERENCES}

[1] I. Csiszár, "On the capacity of noisy channel with arbitrary signal costs," Probl. Control and Inf. Theory, vol. 2, pp. 283-304, 1973.

[2] M. V. Burnashev, "Data transmission over a discrete channel with feedback," Probl. Inf. Trans., vol. 12, no. 4, pp. 10-30, 1976.

[3] H. Yamamoto and K. Itoh, "Asymptotic performance of a modified Schalkwijk-Barron scheme for channels with noiseless feedback," IEEE Trans. Inf. Theory, vol. IT-25, pp. 729-733, November 1979.

[4] T. M. Cover and J. Thomas, Elements of Information Theory. New York: Wiley, 1991.

[5] D. Williams, Probability with Martingales. Cambridge: Cambridge University Press, 1991.

[6] I. Csiszár and J. Körner, Information Theory: Coding Theorems for Discrete Memoryless Systems. New York: Academic Press, 1981. 